\title{
Four Inducible Promoters for Controlled Gene Expression in the Oleaginous Yeast Rhodotorula toruloides
}

\author{
Alexander M. B. Johns, John Love and Stephen J. Aves* \\ Department of Biosciences, University of Exeter, Exeter, UK
}

Rhodotorula (Rhodosporidium) toruloides is an oleaginous yeast with great biotechnological potential, capable of accumulating lipid up to $70 \%$ of its dry biomass, and of carotenoid biosynthesis. However, few molecular genetic tools are available for manipulation of this basidiomycete yeast and its high genomic GC content can make routine cloning difficult. We have developed plasmid vectors for transformation of $R$. toruloides which include elements for Saccharomyces cerevisiae in-yeast assembly; this method is robust to the assembly of GC-rich DNA and of large plasmids. Using such vectors we screened for controllable promoters, and identified inducible promoters from

\section{OPEN ACCESS}

Edited by: Michael H. Perlin,

University of Louisville, USA

Reviewed by:

Lois Hoyer,

University of Illinois

at Urbana-Champaign, USA

Simon Andrew Johnston,

University of Sheffield, UK

*Correspondence:

Stephen J. Aves

s.j.aves@exeter.ac.uk

Specialty section: This article was submitted to Fungi and Their Interactions,

a section of the journal

Frontiers in Microbiology

Received: 12 August 2016 Accepted: 05 October 2016 Published: 21 October 2016

Citation:

Johns AMB, Love $J$ and Aves SJ (2016) Four Inducible Promoters

for Controlled Gene Expression in the Oleaginous Yeast Rhodotorula toruloides. Front. Microbiol. 7:1666.

doi: 10.3389/fmicb.2016.01666 the genes NAR1, ICL1, CTR3, and MET16. These four promoters have independent induction/repression conditions and exhibit different levels and rates of induction in $R$. toruloides, making them appropriate for controllable transgene expression in different experimental situations. Nested deletions were used to identify regulatory regions in the four promoters, and to delimit the minimal inducible promoters, which are as small as 200 bp for the NAR1 promoter. The NAR1 promoter shows very tight regulation under repressed conditions as determined both by an EGFP reporter gene and by conditional rescue of a leu2 mutant. These new tools facilitate molecular genetic manipulation and controllable gene expression in $R$. toruloides.

Keywords: Rhodotorula toruloides, inducible promoters, NAR1, ICL1, CTR3, MET16, in-yeast assembly, G418 resistance

\section{INTRODUCTION}

Rhodotorula toruloides (formerly Rhodosporidium toruloides; Wang et al., 2015) is a red, oleaginous, basidiomycete yeast. It can accumulate lipid up to $70 \%$ of its dry biomass and metabolize a variety of low cost carbon sources, which makes it of interest for biofuel production (Kosa and Ragauskas, 2011; Wiebe et al., 2012). Other proposed uses of this organism include production of carotenoids or other high value chemicals (Lee et al., 2014), as a biocontrol agent (Buck and Andrews, 1999) and as a source of phenylalanine ammonia-lyase for treatment of phenylketonuria (Gilbert and Tully, 1982).

Although $R$. toruloides has great biotechnological potential, as a basidiomycete it is distantly related to more commonly employed yeast such as Saccharomyces cerevisiae. Also, R. toruloides has a high genomic GC content (62\%; Kumar et al., 2012) and consequently a strong bias in its codon usage, necessitating codon optimization of protein coding transgenes (Liu et al., 2013). As a result, 
molecular genetic tools developed for other fungi are rarely directly transferable to $R$. toruloides. In order to perform routine molecular genetic manipulations in this organism, a new toolset must be developed.

Inducible promoters are required for controllable expression of heterologous genes in $R$. toruloides. Several constitutive promoters have been isolated from $R$. toruloides and used to express transgenes, including promoters from the genes GPD1, FBA1, PGK1, PGI1 and TPI1 (Liu et al., 2013; Wang et al., 2016). There is, however, a paucity of inducible promoters characterized for use in $R$. toruloides. The recently isolated DAO1 promoter is strongly induced when $\mathrm{D}$-amino acids are provided as a carbon source, however, it cannot be completely repressed which is a disadvantage for expression of proteins which impede cell growth (Liu et al., 2015). In addition the D-amino acids required for induction are expensive and may be prohibitive for large, industrial fermentations. Finally, induction or repression conditions may affect the results of an experiment. Therefore it would be beneficial to have more than one regulatable promoter available for use in $R$. toruloides.

In this study we extend the range of molecular tools for expression of genes in the oleaginous yeast $R$. toruloides. A system based on in-yeast assembly has been designed for construction of plasmids for transformation of $R$. toruloides using selectable markers including G418 resistance. Potential inducible promoters were screened using an EGFP reporter and we identify and characterize a toolset of four controllable promoters with different induction/repression conditions for use in $R$. toruloides.

\section{MATERIALS AND METHODS}

\section{Strains and Media}

Rhodotorula toruloides wild-type haploid strain CBS 14 (MATA1; ATCC 10788, IFO 0559, MTCC 457; (Rennerfelt, 1937)) and NCYC 1585 (MAT-A2 leu2- ino; (Tully, 1985)), were obtained from the Centraalbureau voor Schimmelcultures, Utrecht, The Netherlands and The National Collection of Yeast Cultures, Norwich, UK, respectively. NCYC 1585 is a mutant derivative of wild-type haploid strain CBS 349 (MAT-A2; ATCC 10657, IFO 0880). $R$. toruloides was grown at $30^{\circ} \mathrm{C}$ in YPD (Sambrook and Russell, 2001), or Yeast Nitrogen Base without amino acids (YNB; ForMedium, Hunstanton, UK) with $20 \mathrm{~g} \mathrm{~L}^{-1}$ glucose. Promoter induction and repression media were YNB with $20 \mathrm{~g} \mathrm{~L}^{-1}$ glucose modified as follows: for SGA1 induction medium, glucose was replaced with maltose; for ICL1 and ICL2 induction medium glucose was replaced with $200 \mathrm{mM}$ sodium acetate; for NAR1 induction medium, YNB without ammonium sulfate was supplemented with $0.78 \mathrm{~g} \mathrm{~L}^{-1}$ potassium nitrate; for THI5 and THI4 induction medium YNB without thiamine was used and for repression medium $20 \mathrm{mg} \mathrm{L}^{-1}$ thiamine was included; for MET16, $1 \mathrm{mM}$ methionine was included in repression medium; for CCC2 induction medium contained $20 \mu \mathrm{M} \mathrm{CuSO}_{4}$ and repression medium was formulated without copper; for CTR3 and CTR31 initial screens induction medium was formulated without copper and repression medium contained $20 \mu \mathrm{M} \mathrm{CuSO}$, for time course and promoter cut-down experiments induction medium contained $100 \mu \mathrm{M}$ bathocuproinedisulfonic acid (BCS). Solid media contained 2\% agar except for NAR1 induction/repression media where $2 \%$ agarose was used.

Agrobacterium tumefaciens strain GV3101 (van Larebeke et al., 1974) was grown at $28^{\circ} \mathrm{C}$ in LB (Cold Spring Harbor, 2006) containing rifampicin $\left(50 \mu \mathrm{g} \mathrm{mL}{ }^{-1}\right)$. Cloning was performed using Escherichia coli NEB5 $\alpha$ (New England Biolabs, Ipswich, $\mathrm{MA}$, usa) grown in $\mathrm{LB}$ at $37^{\circ} \mathrm{C}$. In-yeast assembly was performed

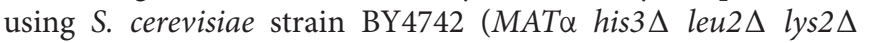
ura3 $\Delta$; Brachman et al., 1998) grown in YPD, or YNB with $20 \mathrm{~g} \mathrm{~L}^{-1}$ glucose and Complete Supplement Mix without uracil (ForMedium, Hunstanton, UK) for auxotrophic selection.

\section{General Molecular Biological Techniques}

Chemicals were supplied by Sigma-Aldrich (St Louis, MO, USA) unless indicated. Synthetic DNA was produced by GeneArt (ThermoFisher, Waltham, MA, USA). DNA manipulation was performed using standard techniques (Sambrook and Russell, 2001). PCR was performed using Q5 polymerase (New England Biolabs, Ipswich, MA, USA) with oligonucleotides purchased from Eurofins (Ebersberg, DE). Plasmid DNA was prepared from E. coli by alkaline lysis using a GeneJET Plasmid Miniprep Kit (ThermoFisher, Waltham, MA, USA). Restriction digests were performed using High-Fidelity restriction endonucleases (New England Biolabs, Ipswich, MA, USA). All cloning was verified by Sanger sequencing (Source Biosciences, Nottingham, UK).

E. coli was chemically transformed using High Efficiency Transformation (New England Biolabs, Ipswich, MA, USA). Chemically competent $A$. tumefaciens was prepared and transformed by the protocol of Holsters et al. (1978).

\section{S. cerevisiae In-yeast Assembly}

In-yeast assembly was performed by a modified version of the protocol of Kilaru and Steinberg (2015). Briefly, DNA fragments with overlapping homology regions of $25 \mathrm{bp}$ at their ends were co-transformed into $S$. cerevisiae in an approximately equimolar ratio using a yeast transformation kit (Sigma-Aldrich, St Louis, MO, USA). In-yeast assembled plasmids were extracted by the method of Singh and Weil (2002), transformed into E. coli, isolated by alkaline lysis and verified by Sanger sequencing of junctions.

\section{Plasmid Construction}

Plasmid pG418-Rt was constructed using Gibson assembly (Gibson et al., 2009). pCAMBIA0380 (Cambia, Canberra, ACT, Australia) was digested using PvuI; the $R$. toruloides CBS 14 GPD1 promoter was amplified from genomic DNA using primers RtGPD1F-pCambia0380 and GPD1R-G418 (Table 1). These two components were assembled with a codon-optimized $\operatorname{APH}\left(3^{\prime}\right)$ G418 resistance gene amplified using primers G418F-GPD1 and G418R-pCambia0380, using an NEB Gibson Assembly Cloning Kit (New England Biolabs, Ipswich, MA, USA).

pEGFP-Rt-YR-G418 was constructed in two steps by in-yeast assembly of plasmid pC-G418-YR (Sidhu et al., 2015) digested with $P v u I I$, and codon-optimized G418 resistance gene under regulation of the GPD1 promoter amplified from pG418-Rt 
TABLE 1 | Primers used.

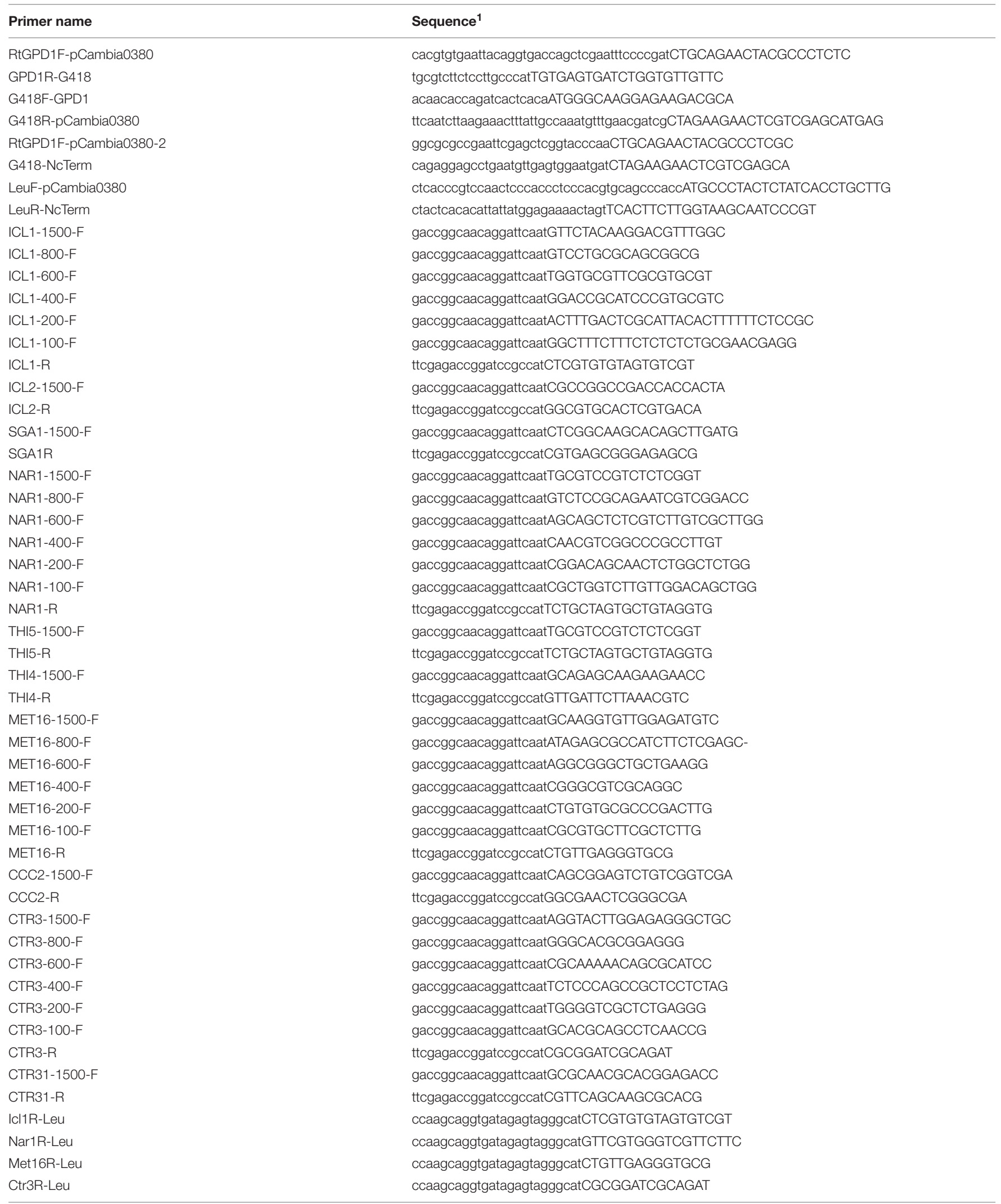

${ }^{1}$ Priming sequences are shown in uppercase and $5^{\prime}$ extensions in lowercase. 
using primers RtGPD1F-pCambia0380-2 and G418-NcTerm; the resulting plasmid was digested using HindIII and assembled in-yeast with synthetic DNA comprising the $R$. toruloides PGK1 promoter, codon-optimized EGFP gene, and CMV35S terminator.

Plasmids for testing promoter activity were constructed by in-yeast assembly of AflII/PmlI-digested pEGFP-Rt-YR-G418 with promoter fragments amplified from genomic DNA using respective primers (Table $\mathbf{1}$ ).

Plasmid pLeu-Rt-YR-G418 was constructed by amplification of the $R$. toruloides CBS 14 LEU2 gene using primers LeuFpCambia0380 and LeuR-NcTerm, and in-yeast assembly with pEGFP-Rt-YR-G418 digested with PmlI and SpeI. Plasmids for conditional leu2 $2^{-}$rescue under the regulation of ICL1, NAR1, MET16 and CTR3 1500-bp promoter fragments were assembled in the same manner as plasmids for testing promoter activity with EGFP, with the modifications that pLeu-Rt-YR-G418 was used instead of pEGFP-Rt-YR-G418 as the base plasmid, and reverse primers Icl1R-Leu, Nar1R-Leu, Met16R-Leu and Ctr3RLeu were used for amplification of ICL1, NAR1, MET16 and CTR3 promoters, respectively.

\section{Transformation of $\boldsymbol{R}$. toruloides}

Transformation of $R$. toruloides was performed using a modified version of the protocol of Liu et al. (2013). A. tumefaciens containing the appropriate binary plasmid was grown in LB with rifampicin $\left(50 \mu \mathrm{g} \mathrm{mL}^{-1}\right)$ and kanamycin $\left(50 \mu \mathrm{g} \mathrm{mL}^{-1}\right)$ at $28^{\circ} \mathrm{C}$ for $48 \mathrm{~h}$, then diluted to an $\mathrm{OD}$ of approximately 0.1 in induction medium (Gelvin, 2006) at $24^{\circ} \mathrm{C}$ for $6 \mathrm{~h}$. A $200 \mu \mathrm{L}$ volume of this A. tumefaciens culture was then mixed with $200 \mu \mathrm{L}$ of an overnight culture of $R$. toruloides, spread over a nitrocellulose membrane on solid induction medium and incubated at $24^{\circ} \mathrm{C}$ for $48 \mathrm{~h}$. Membranes were transferred to YPD with G418 (150 $\mu \mathrm{g}$ $\left.\mathrm{mL}^{-1}\right)$ and cefotaxime $\left(150 \mu \mathrm{g} \mathrm{mL}^{-1}\right)$ and incubated at $30^{\circ} \mathrm{C}$ for 2-3 days. Colonies were restreaked to fresh selective YPD and grown overnight.

\section{Measurement of EGFP Expression}

For initial screening and promoter cut-down experiments three independent transformants were each grown overnight in YNB, pelleted by centrifugation $(2500 \times g$ for $5 \mathrm{~min})$ and washed twice with sterile water. Approximately $10^{7}$ cells were added to $20 \mathrm{~mL}$ induction/repression medium and allowed to grow for $16 \mathrm{~h}$ ( $8 \mathrm{~h}$ for MET16 promoter cut-down experiments). Samples of $0.5 \mathrm{ml}$ were then taken and kept on ice until fluorescence could be measured. For measurement of induction rates, starter cultures were grown overnight in repressive conditions. Cells were then pelleted by centrifugation and washed twice with sterile water. Approximately $10^{7}$ cells were added to $50 \mathrm{~mL}$ induction or repression medium and grown at $30^{\circ} \mathrm{C}$. Samples of $0.5 \mathrm{~mL}$ were taken at the indicated time intervals and kept on ice until fluorescence could be measured.

Fluorescence was quantified by flow cytometry using a FACSAria II (BD Biosciences, San Jose, CA, USA) with excitation at $488 \mathrm{~nm}$ and a $530 / 30 \mathrm{~nm}$ emission filter. To quantify cell density, CountBright absolute counting beads (ThermoFisher, Waltham, MA, USA) were added to samples. Data were analyzed using FlowJo software (FlowJo, Ashland, OR, USA) to determine median fluorescence for each sample. Student's $t$-tests were conducted to determine statistical significance between different experimental conditions.

\section{Auxotrophic Rescue}

Cells were grown overnight in induction or repression medium with leucine $\left(100 \mathrm{mg} \mathrm{L}^{-1}\right)$ as indicated, harvested by centrifugation, washed twice, then re-suspended in sterile water to approximately $10^{6}$ cells $\mathrm{mL}^{-1}$. A $10 \mathrm{x}$ serial dilution was then spotted on to solid induction or repression media with or without leucine using a replica plater (Sigma-Aldrich, St Louis, MO, USA).

\section{Motif Discovery}

Motif discovery was performed using MEME, version 4.11.2 hosted at http://meme-suite.org/tools/meme using default settings. Genomes used for comparison were: $R$. toruloides CBS 14 (Kumar et al., 2012), R. toruloides CBS 349 (Zhang et al., 2016), R. graminis WP1 (Firrincieli et al., 2015), Sporobolomyces (formerly Sporidiobolus) salmonicolor CBS 6832 (Coelho et al., 2015), S. roseus JGIBAIF-5F1, Phyllozyma (formerly Sporobolomyces) linderae CBS 7893, Microbotryum lychnidisdioicae p1A1 (Grigoriev et al., 2014), Mixia osmundae IAM 14324 (Toome et al., 2014), Leucosporidium creatinivorum (formerly Leucosporidiella creatinivora; Grigoriev et al., 2014) and Puccinia graminis (Duplessis et al., 2011). Searching for known elements within promoters was performed using FIMO, version 4.11.2 hosted http://meme-suite.org/tools/fimo using default settings.

\section{RESULTS AND DISCUSSION}

\section{Identification of Candidate Inducible Promoters in $\boldsymbol{R}$. toruloides}

To identify a toolset of inducible promoters for use in different situations, we screened potential inducible promoters based on successful use in other fungi. Orthologs of promoters regulated by carbon source, nitrogen source, metabolite availability, and copper availability were identified in the $R$. toruloides CBS 14 haploid genome by reciprocal BLASTP hits against their respective genes, and are listed in Table 2 . This work focused on the $R$. toruloides haploid strain CBS 14 as its lipid production is well characterized (Evans and Ratledge, 1984; Wiebe et al., 2012, Zhang et al., 2016), the genome has been sequenced (Kumar et al., 2012; Zhang et al., 2016), and it is almost identical to strain NP 11 (Zhu et al., 2012; Zhang et al., 2016) which has been the subject of in depth multi-omic study (Zhu et al., 2012). We checked that $R$. toruloides CBS 14 could grow in induction and repression conditions for each candidate promoter. Growth was observed in all media except where galactose was the sole carbon source; as a result GAL1 and GAL7 were excluded from further analysis. 
TABLE 2 | $\boldsymbol{R}$. toruloides candidate inducible promoters.

\begin{tabular}{|c|c|c|c|c|}
\hline Gene $^{1}$ & Predicted protein & Induced by & Repressed by & Reference \\
\hline \multirow[t]{2}{*}{ GAL 1} & Galactokinase & + Galactose & + Glucose & Ruff et al., 2009 \\
\hline & & - Glucose & & \\
\hline \multirow[t]{2}{*}{$G A L 7$} & Galactose-1-phosphate uridyl transferase & + Galactose & + Glucose & \\
\hline & & - Glucose & & \\
\hline \multirow[t]{3}{*}{ SGA1 } & Glucoamylase & + Maltose & + Xylose & Siedenberg et al., 1999 \\
\hline & & + Starch & + Glucose & Aspergillus niger GlaA \\
\hline & & - Glucose & & \\
\hline \multirow[t]{2}{*}{ ICL1 } & Isocitrate lyase 1 & + Acetate & + Glucose & Barth, 1985 \\
\hline & & - Glucose & & \\
\hline \multirow[t]{2}{*}{ ICL2 } & Isocitrate lyase 2 & + Acetate & + Glucose & \\
\hline & & - Glucose & & \\
\hline \multirow[t]{2}{*}{ NAR1 } & Nitrate reductase & + Nitrate & + Ammonium & Banks et al., 1993 \\
\hline & & - Ammonium & & \\
\hline \multirow[t]{2}{*}{ THI5 } & 4-amino-5-hydroxymethyl-2-methylpyrimidine phosphate synthase & - Thiamine & + Thiamine & Maundrell, 1990 \\
\hline & & & & Schizosaccharomyces pombe nmt1 \\
\hline \multirow[t]{2}{*}{ THI4 } & Thiamine thiazole synthase & - Thiamine & + Thiamine & Manetti et al., 1994 \\
\hline & & & & S. pombe nmt2 \\
\hline MET16 & $3^{\prime}$ phosphoadenylsulfate reductase & - Methionine & + Methionine & Solow et al., 2005 \\
\hline \multirow[t]{2}{*}{ CCC2 } & Copper efflux pump & + Copper & - Copper & Gebhart et al., 2006 \\
\hline & & & & Histoplasma capsulatum CRP1 \\
\hline CTR3 & High affinity copper transporter & - Copper & + Copper & Labbé and Thiele, 1999 \\
\hline CTR31 & Copper transporter & - Copper & + Copper & Paralog of CTR3 \\
\hline
\end{tabular}

${ }^{1}$ Gene names reflect S. cerevisiae ortholog.

\section{In-Yeast Assembly for Construction of Vectors}

The high GC content of R. toruloides DNA, or of genes codon optimized for use in $R$. toruloides, reduces the efficiency of in vitro cloning techniques for assembly of plasmids for manipulation of this organism. S. cerevisiae in-yeast assembly is robust to the assembly of large or GC-rich fragments (Agarwal et al., 1970; Noskov et al., 2012) therefore this technique was used for vector construction. In-yeast assembly exploits the high efficiency of homologous recombination in S. cerevisiae to assemble multiple DNA fragments into a circular replicating plasmid (Noskov et al., 2012). The range of selectable markers is limited for R. toruloides so we developed G418 selection for transformation of $R$. toruloides. A cassette consisting of codonoptimized APH $\left(3^{\prime}\right)$ G418 resistance gene under regulation of the $R$. toruloides GPD1 constitutive promoter was found to confer resistance to this antibiotic at a concentration of $150 \mu \mathrm{g} \mathrm{mL}^{-1}$. This G418 resistance marker expands the number of selection markers available for use in $R$. toruloides and uses a cheaper and safer antibiotic compared with the previously used hygromycin, bleomycin, and nourseothricin (Liu et al., 2013; Lin et al., 2014).

Plasmid pC-G418-YR (Kilaru and Steinberg, 2015) Zymoseptoria tritici transformation vector was used as a base for assembly of $R$. toruloides transformation vectors. This plasmid is a derivative of pCAMBIA0380 modified to include a URA3 selection marker and a $2 \mu$ origin of replication in the vector backbone, facilitating maintenance in S. cerevisiae. It also includes a G418 resistance marker regulated by $Z$. tritici $\alpha$-tubulin promoter and Neurospora crassa $\beta$-tubulin terminator in the T-DNA region. We excised the Z. tritici promoter and G418 resistance marker and replaced them with the $R$. toruloides codon-optimized G418 resistance marker under regulation of $R$. toruloides CBS 14 GPD1 constitutive promoter (Liu et al., 2013). We also inserted a synthetic construct into the T-DNA region containing a codon-optimized EGFP gene under regulation of the $R$. toruloides PGK1 promoter (Lin et al., 2014; mutated to include a PmlI cut site at the -7 to -12 position) and the CMV35S terminator. An AflII cut site was incorporated upstream of the promoter and a SpeI site immediately downstream of the EGFP. The resulting plasmid, pEGFP-Rt-YR-G418 (Figure 1A), is designed such that the PGK1 promoter or the EGFP gene can easily be exchanged by digestion with AflII/PmlI or PmlI/SpeI, respectively, and the promoter or gene of interest inserted by in-yeast assembly (Figure 1B). A second variant (pEGFP-Rt-YR-Hyg) was produced replacing the G418 resistance gene with a codon-optimized hygromycin resistance marker (Liu et al., 2013).

To test each of the 10 promoters, $1500 \mathrm{bp}$ upstream of the translational start site was amplified by PCR and inserted in place of the PGK1 promoter, upstream of the EGFP reporter gene (Figure 1B).

\section{GFP Screening Identifies NAR1, ICL1, CTR3 and MET16 Inducible Promoters in R. toruloides}

Each promoter-EGFP construct was transformed into R. toruloides haploid strain CBS 14 . To identify which candidate promoters can be used as regulatable promoters, cultures were 


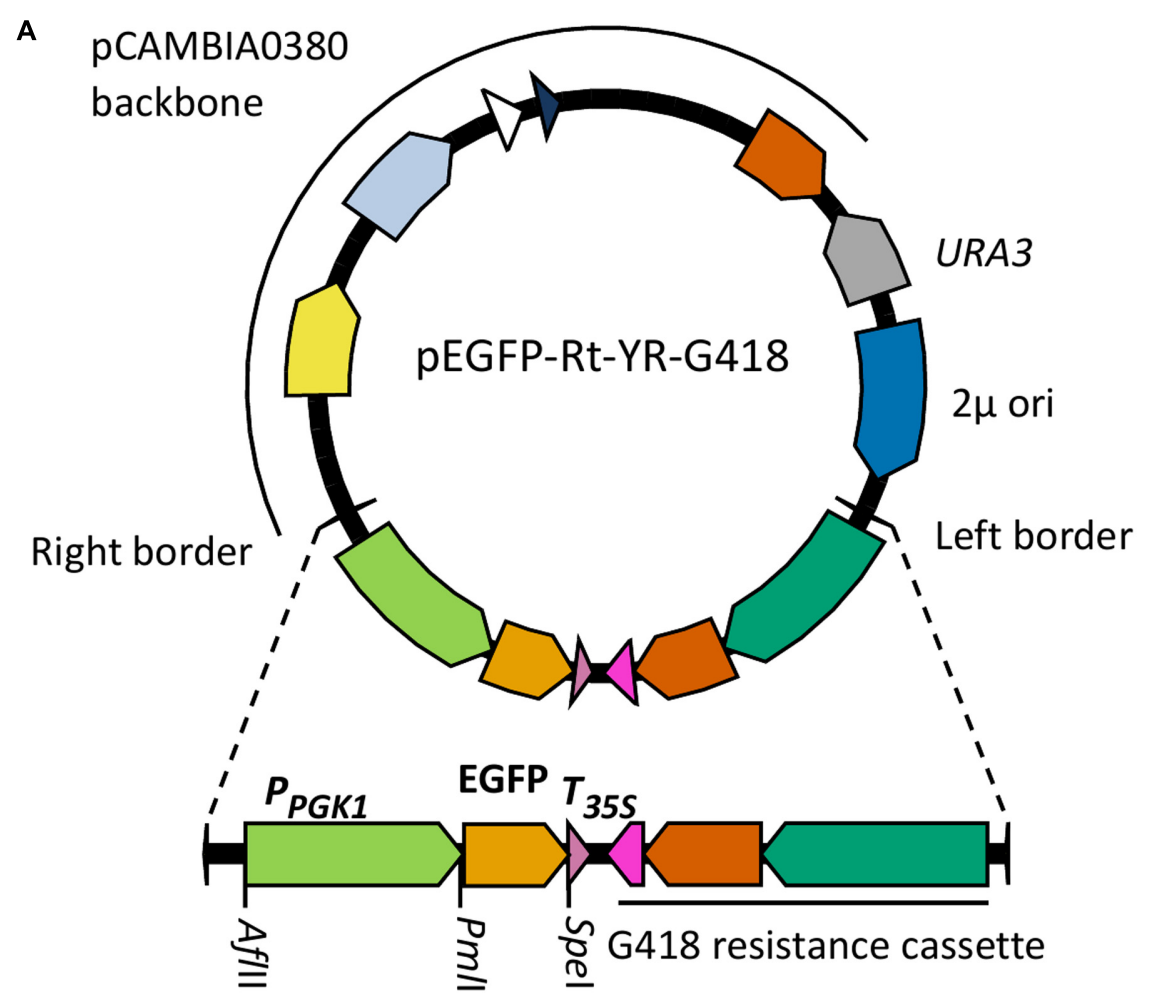

B

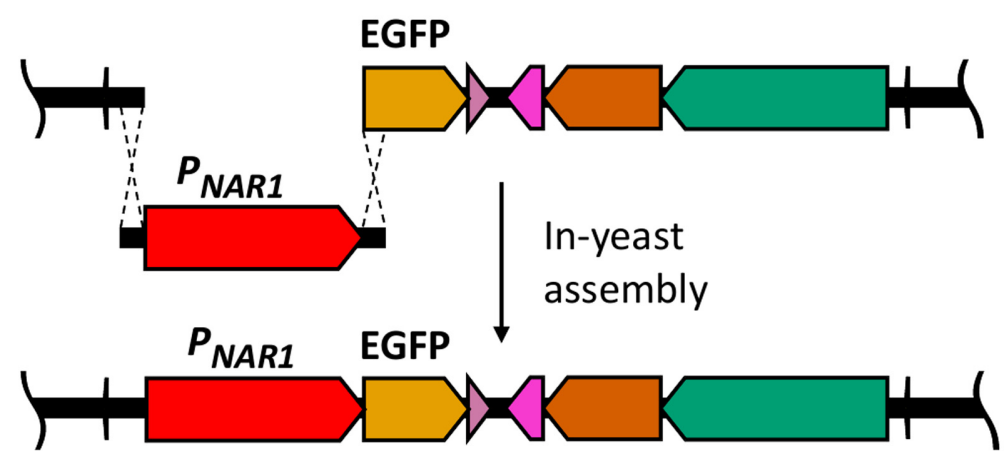

FIGURE 1 | Cloning strategy for identification of inducible promoters in $\boldsymbol{R}$. toruloides. (A) R. toruloides transformation vector pEGFP-Rt-YR-G418. The vector incorporates a URA3 marker and $2 \mu$ origin for maintenance in $S$. cerevisiae. The T-DNA region to be integrated into the $R$. toruloides genome is shown expanded; the $R$. toruloides G418 resistance cassette consists of a codon-optimized APH( $\left.3^{\prime}\right)$ gene (orange) under regulation of the $R$. toruloides GPD1 promoter (green) and N. crassa beta tubulin terminator (pink). The pCAMBIA0380 backbone contains, in a clockwise direction: the right border sequence (RB); pVS1 StaA stability region (yellow) and RepA replication origin (light blue) for maintenance in A. tumefaciens; pBR322 bom (white) and ori (dark blue) for maintenance in E. coli; kanMX kanamycin resistance cassette (orange). pCAMBIA0380 also provides the left border sequence (LB). (B) Cloning strategy for inserting promoters of interest upstream of EGFP gene. The promoter of interest, the NAR1 promoter in the example shown, is amplified with 25-bp overhangs complementary to regions flanking the insertion site. This is co-transformed into S. cerevisiae along with pEGFP-Rt-YR-G418 pre-digested with Aflll and Pm/l. In vivo homologous recombination inserts the promoter upstream of the EGFP gene in the vector.

grown for $16 \mathrm{~h}$ under induced and repressed conditions and EGFP fluorescence measured by flow cytometry. To minimize any positional effects from the locus of integration of the T-DNA into the $R$. toruloides genome, each test was performed on three independently transformed biological replicates.

Of the candidates screened, the promoters of ICL1, NAR1, and MET16 demonstrated inducibility (Figure 2A). The NAR1 promoter displayed high levels of induced expression surpassed only by the THI5 and THI4 constitutive promoters. This promoter also exhibited low expression when repressed (measured induction ratio $=29$ ).

The ICL1 promoter also displayed high levels of induced expression; however, ICL1 repression was incomplete in the presence of glucose (measured induction ratio $=7.6$ ). This is consistent with activity observed in the oleaginous ascomycete yeast Yarrowia lipolytica as well as the economically important 

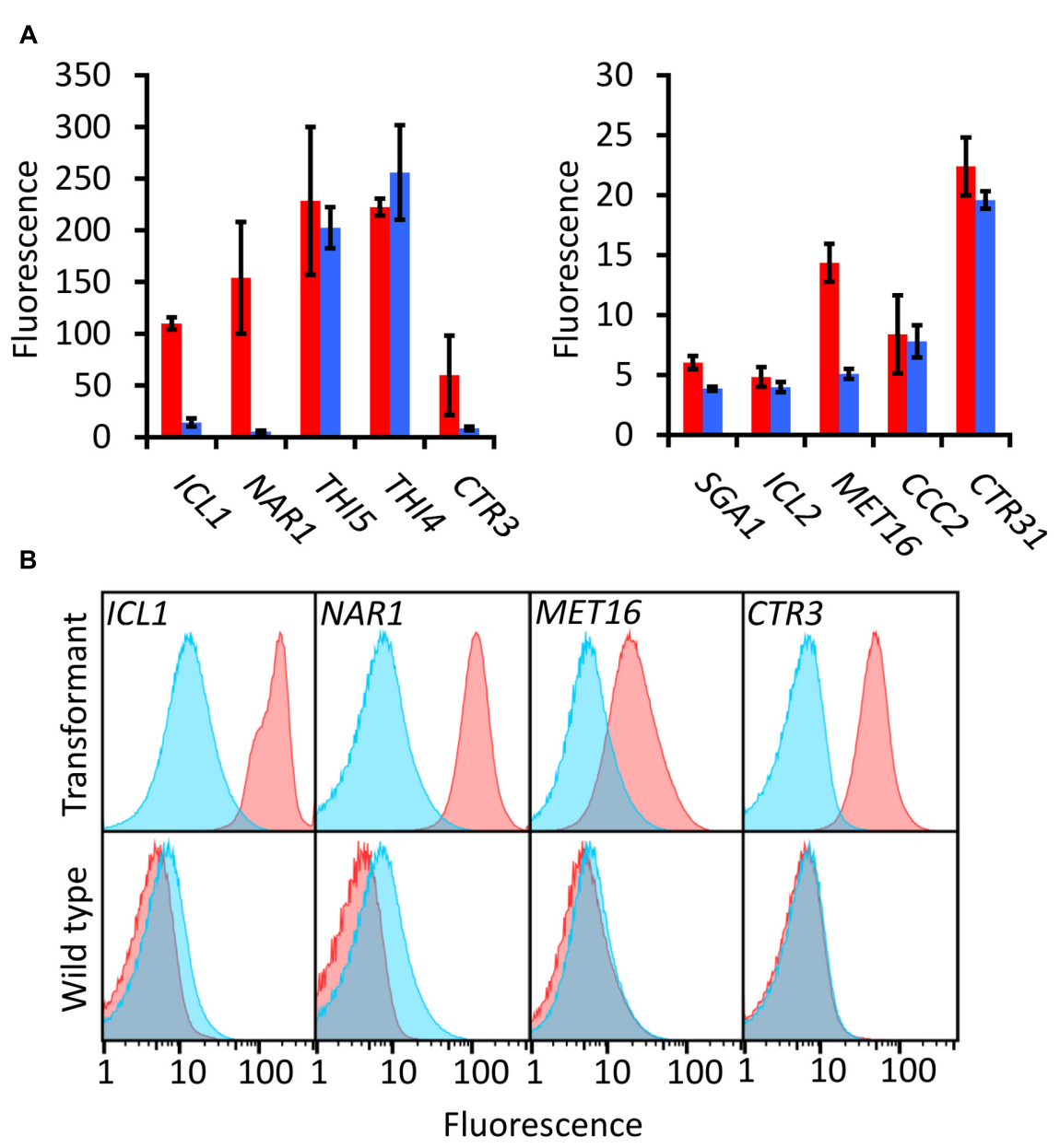

FIGURE 2 | EGFP-based screening of ten candidate inducible promoters in $\boldsymbol{R}$. toruloides. (A) Fluorescence of $R$. toruloides cells with EGFP regulated by test promoters after overnight growth in induced conditions (red bars) and repressed conditions (blue bars). Fluorescence was measured by flow cytometry and the median cellular fluorescence determined for $\geq 500000$ cells per culture. Bars indicate the mean of three independently transformed biological replicates with the standard deviation shown as error bars. Induction and repression mean values are significantly different $(p<0.05)$ for promoters ICL1, NAR1, SGA1 and MET16, as determined by student's $t$-test. (B) Representative histograms showing fluorescence of cells in induced (red) and repressed (blue) conditions for the ICL 1 , NAR1, MET16 and CTR3 promoters. Upper panels show transformant cells with EGFP under the regulation of each indicated promoter; lower panels show autofluorescence of untransformed cells under growth conditions identical to the transformants above.

Komagataella (formerly Pichia) pastoris (Barth, 1985; Menendez et al., 2003). Acetic acid has been proposed as a feedstock for industrial growth of $R$. toruloides due to its low cost (Huang et al., 2016), and under these conditions the ICL1 promoter would be induced. Such a system has been proposed for protein production in $K$. pastoris, as an alternative to the commonly used methanol-induced $A O X$ promoter (Menendez et al., 2003).

The MET16 promoter had a low induced expression level (about one tenth the strength of the induced NAR1 promoter) and also a low induction ratio. However, under repressed conditions the measured fluorescence was comparable to the autofluorescence of untransformed cells under identical conditions (Figure 2B), therefore the apparent induction ratio of 2.8 should be considered a minimum.

The CTR3 promoter exhibited strong repression in the presence of copper and had a medium level of induction in its absence; however, there was a large degree of variation between the replicates. For this reason the copper chelator BCS was added to induction medium in all subsequent experiments; this resulted in consistent and significant induction of the CTR3 promoter. The NAR1 and ICL1 promoters require changes in nitrogen or carbon sources, respectively, between induced and repressed conditions; this would have effects on global metabolism whereas the copper starvation conditions for induction of the CTR3 promoter are unlikely to lead to such gross changes in metabolism (Ouyang et al., 2015). The CTR3 inducible promoter can therefore be useful where background metabolic considerations are important, such as in a laboratory setting.

Other promoters screened either showed constitutive activity (THI5, THI4, CTR31) or little to no induced fluorescence under the conditions tested (SGA1, ICL2 and CCC2). 


\section{Gene Expression Is Activated within 4-16 h of Promoter Induction}

The rate of induction for each of the four promoters was measured by performing a time course over $24 \mathrm{~h}$ from transfer to induction medium, after overnight culture in repression medium (Figure 3). Autofluorescence due to carotenoids produced during late log and stationary phase gives high background after $24 \mathrm{~h}$ making measurements unreliable (Kleinegris et al., 2010; Lee et al., 2014).

The MET16 promoter was the fastest to induce, reaching a maximum after $4 \mathrm{~h}$ and declining after $8 \mathrm{~h}$. This promoter may therefore be suitable for experiments where rapid induction is desirable but high-level expression is not required. Both the NAR1 and ICL1 promoters showed greatest increases in expression after $8 \mathrm{~h}$, reaching maxima at around $16 \mathrm{~h}$. In the presence of the copper chelator BCS, induction of the CTR3 promoter started at $2 \mathrm{~h}$ and increased asymptotically up to $24 \mathrm{~h}$ (Figure 3).

Cultures with sodium acetate as the sole carbon source grew slowly relative to cultures with glucose. In a laboratory setting this may be problematic when comparing the biology of cultures in induced and repressed conditions for the ICL1 promoter, and in an industrial setting may cause reduction in yield; however, this could be overcome by using a two-stage fermentation, initially growing with glucose and then switching to growth on acetate.
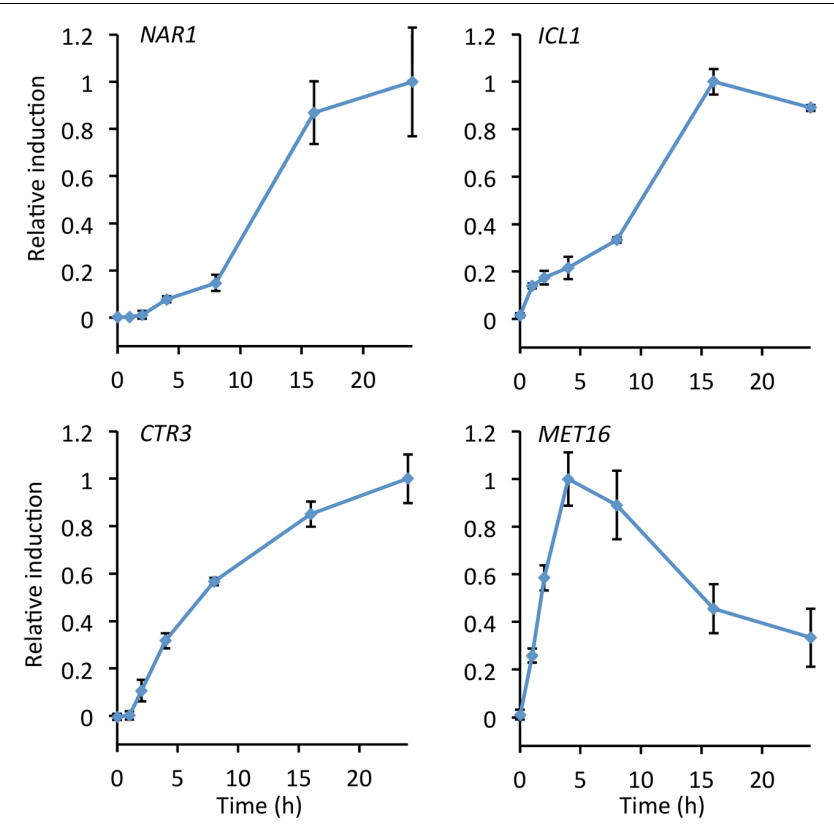

FIGURE 3 | Time course showing relative promoter induction up to $\mathbf{2 4} \mathbf{h}$ after transfer to inducing conditions. Cultures were grown in repressive medium overnight before cultures were washed, split, and transferred to fresh induction/repression medium. Samples were taken at the times indicated and fluorescence measured. Induction was calculated as fluorescence under induced conditions minus fluorescence under repressed conditions and normalized to maximum observed induction. Points show the mean of three independently transformed biological replicates; error bars indicate standard deviation.

\section{Conditional Mutant Rescue Using the NAR1 Promoter}

To investigate controllable mutant rescue the $R$. toruloides leu2 mutant strain NCYC 1585 was used (Tully, 1985; Lin et al., 2012). The EGFP gene in vector pEGFP-Rt-YR-G418 was replaced by LEU2 from $R$. toruloides CBS 14 to give plasmid pLeu-Rt-YRG418. This construct rescued $R$. toruloides NCYC 1585 growth on leucine deficient medium; transformants could be selected either by growth on leucine-minus medium or by G418 resistance.

The promoter driving the LEU2 gene was then exchanged for each of the four inducible promoters and these constructs transformed into $R$. toruloides NCYC 1585, selecting for transformants with G418. Transformant strains were grown overnight in induction media supplemented with leucine and spot plated to solid induction/repression media with or without leucine. All transformants were able to grow under induction conditions in the absence of leucine, indicating mutant rescue by LEU2 under the transcriptional control of each of the four inducible promoters. On solid medium under repressive conditions, transformants carrying LEU2 under the regulation of the NAR1 promoter were unable to grow (Figure 4) demonstrating conditional rescue of leu2 $R$. toruloides using the NAR1 promoter, and confirming low expression levels under repressive conditions for this promoter.

Cells transformed with LEU2 under regulation of ICL1, CTR3, or MET16 promoters were able to grow under repressive conditions indicating incomplete repression (Supplementary Figure 1). This could reflect strain differences, as the NCYC

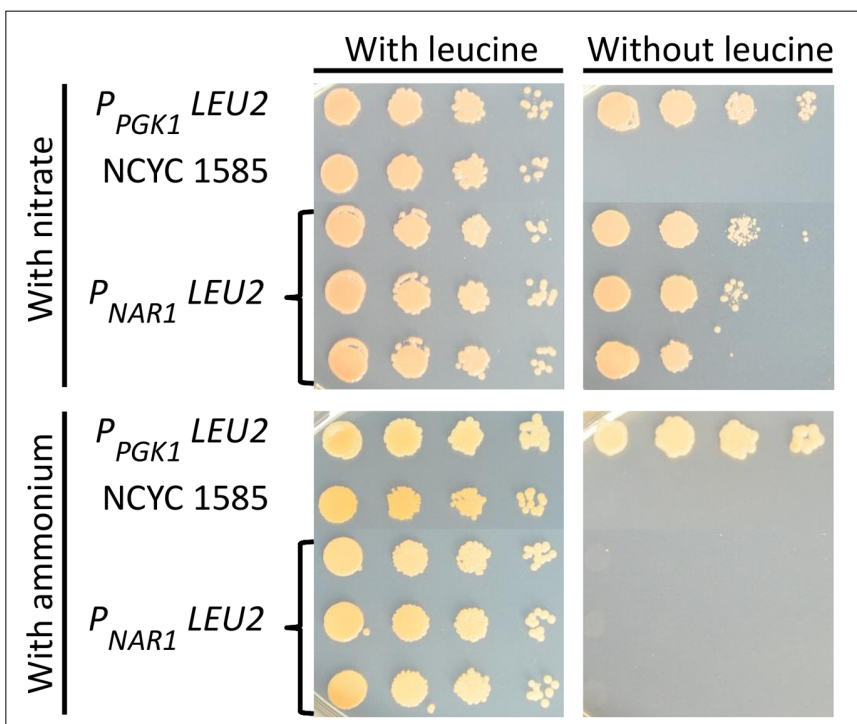

FIGURE 4 | Conditional rescue of leu2 mutant $R$. toruloides strain NCYC 1585 with LEU2 under regulation of the NAR1 promoter. Cells from three independent transformant lines were grown overnight in induction medium with leucine (100 $\mathrm{mg} \mathrm{L}^{-1}$ ) and plated on to YNB with $2 \%$ agarose with either $3.5 \mathrm{~g} \mathrm{~L}^{-1}$ ammonium sulfate or $0.78 \mathrm{~g} \mathrm{~L}^{-1}$ potassium nitrate and allowed to grow for 4 days. Cells transformed with LEU2 under the regulation of the constitutive PGK1 promoter and untransformed NCYC 1585 cells were included as positive and negative controls, respectively. 


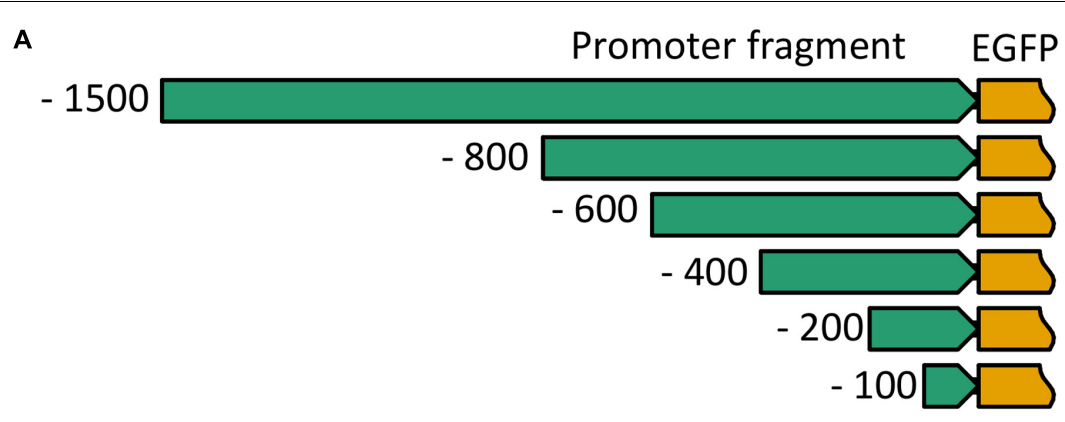

B

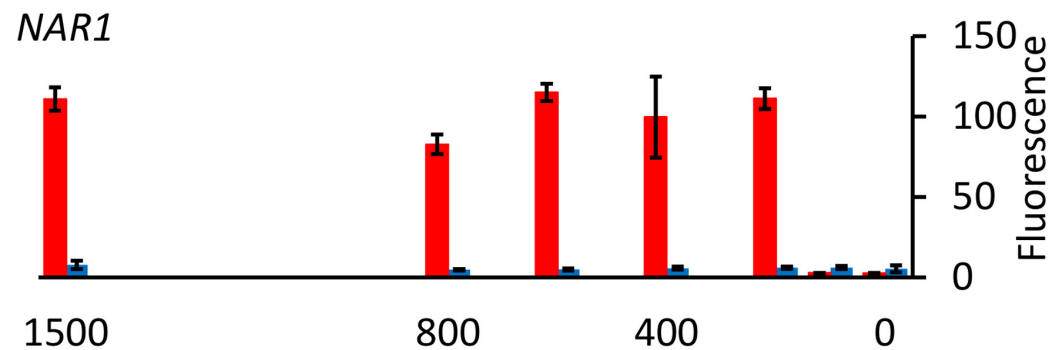

ICL1

C

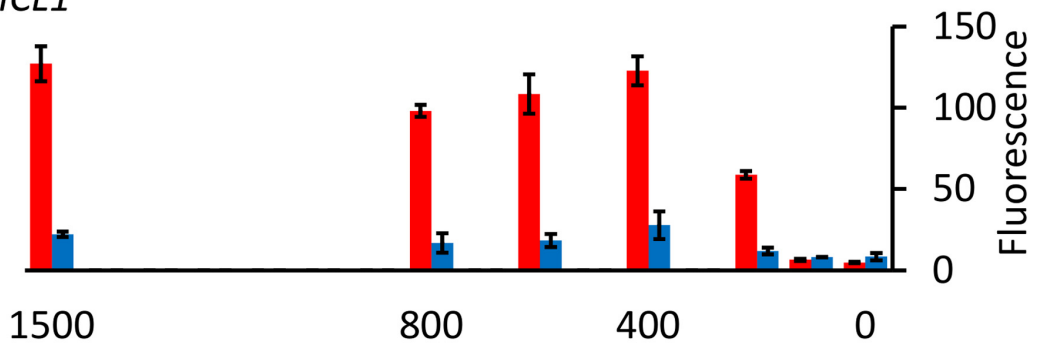

CTR3

D
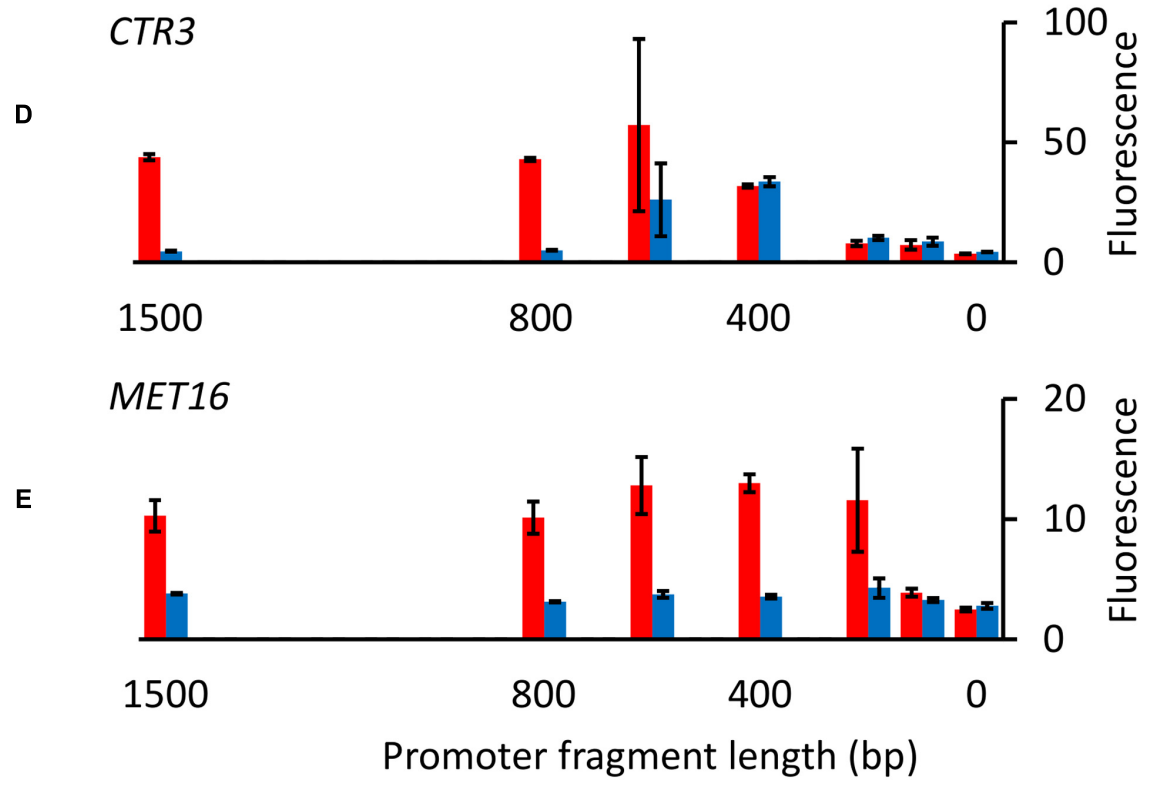

FIGURE 5 | Nested deletion of promoter fragments. (A) Diagram of constructs produced. For each of the four inducible promoters fragments 100, 200, 400, 600, 800, and 1500 bp in length upstream from the ATG were cloned upstream of the codon-optimized EGFP gene. (B-E) EGFP fluorescence of cells carrying truncated versions of NAR1 (B), ICL1 (C), CTR3 (D) and MET16 (E) promoters under induced (red) and repressed (blue) conditions. Cells were grown overnight and then transferred to fresh induction/repression media. Fluorescence was measured after $16 \mathrm{~h}$ growth in induction/repression media (8 $\mathrm{h}$ for MET16). Bars show the mean fluorescence of three independently transformed biological replicates; error bars indicate standard deviation. Induction and repression mean values are significantly different $(p<0.05)$ for the following promoter fragment lengths: NAR1 $\geq 200 \mathrm{bp}$; ICL1 $\geq 200 \mathrm{bp}$; CTR3 $\geq 800 \mathrm{bp} ;$ MET16 $\geq 400 \mathrm{bp}$ and $100 \mathrm{bp}$. 
1585 leu2 strain is a derivative of $R$. toruloides strain CBS 349 which shares only $87 \%$ DNA sequence identity with CBS 14 (Kumar et al., 2012; Zhang et al., 2016), although the two strains can mate (Banno, 1967). Alternative explanations are possible, for example regulatory elements within a LEU2 intron enhancing promoter expression, as in the case of the DAO1 promoter in strain CBS 349 (Liu et al., 2015), but this would require further study to explore.

\section{Functional Dissection of $\boldsymbol{R}$. toruloides Inducible Promoters}

Initially promoter fragments tested were all 1500 bp in length. To identify the minimum size of each promoter required for controllable gene expression and the location of regulatory elements, nested deletions of each of the four inducible promoters were cloned upstream of the EGFP gene (Figure 5A) and fluorescence measured for $R$. toruloides CBS 14 transformants under induced and repressed conditions.

With the NAR1 promoter no activity was observed with the 100-bp fragment but full regulation was observed with fragments $200 \mathrm{bp}$ and longer (Figure 5B), demonstrating all necessary controlling elements are present in this short region. Similarly, for the ICL1 and MET16 promoters, little or no activity was observed with the 100-bp fragments, full regulation required 400bp fragments, with 200 bp giving partial activity under induced conditions for ICL1 (Figures 5C,E). CTR3 promoter cut-downs showed a more interesting pattern: 100- and 200-bp fragments showed little activity, the 400-bp fragment was constitutively active, and the 800-bp and 1500-bp fragments exhibited full regulation (Figure 5D).

To identify functional elements within essential promoter regions, a motif search was performed using MEME for conserved elements between orthologous promoters in $R$. toruloides and related members of the Pucciniomycotina. In both the ICL1 and CTR3 promoters, CT-rich boxes were identified in the -50 to -40 region relative to the start codon. Similar elements have been observed in the R. toruloides GPD1 and DAO1 promoters (Liu et al., 2013, 2015) indicating this is a highly conserved element in $R$. toruloides. Such an element has also been observed in other filamentous fungi where it is proposed to be responsible for targeting the translational start site (Punt et al., 1990).

In the CTR3 promoter a second conserved box was identified at -583 to -602 with the consensus GCRAAAANNGCGCATC. The 400-bp promoter fragment showed constitutive induction, the 600-bp fragment exhibited variable repression and the 800bp fragment full repression in the presence of copper; this sequence element could therefore be responsible for repression of this promoter in the presence of copper. Other instances of this element were identified in $R$. toruloides promoters using FIMO (Grant et al., 2011) and the genes adjacent to the top 10 hits identified. Apart from CTR3, the top hit was upstream of a vacuolar ABC heavy metal transporter, a gene likely to be regulated by copper, and the second hit was in the promoter for salicylate hydroxylase, the product of which (catechol) is toxic in the presence of heavy metals (Schweigert et al., 2001) and thus would likely be repressed in the presence of copper. The motif was also identified 283 bp downstream of a second gene annotated as a copper transporter. Given the range at which this element acts it is possible that this element can act on the promoter of this gene from this location.

\section{CONCLUSION}

We have characterized four inducible promoters to allow controllable expression in the oleaginous yeast $R$. toruloides, designed vectors for efficient cloning of its high-GC DNA, and added to the range of useful selectable markers for this yeast. The NAR1 promoter is strongest when induced, shows tight regulation under repressed conditions in two $R$. toruloides strain backgrounds, has a short $200 \mathrm{bp}$ functional sequence, and would be the first choice promoter in many cases. However, each promoter has its own individual characteristics that render it suitable for particular applications, and together they provide a suite of complementary regulatory elements for controlling gene expression in this yeast.

\section{AUTHOR CONTRIBUTIONS}

Experiments were conceived and designed by AJ and SA with assistance from JL. Experiments were performed by AJ. Analysis was performed by AJ and SA with assistance from JL. SA and JL supervised the project. The manuscript was written by AJ and SA with assistance from JL.

\section{FUNDING}

This work was supported by Biotechnology and Biological Sciences Research Council doctoral training partnership award BB/J014400/1 to AJ.

\section{ACKNOWLEDGMENTS}

We would like to thank Yaadwinder Sidhu for supplying plasmid pC-G418-YR and assistance with in-yeast assembly, Sreedhar Kilaru for help in selection of potential promoters, and Sebastian Bowyer and George Kirke for assistance with promoter cut-down experiments.

\section{SUPPLEMENTARY MATERIAL}

The Supplementary Material for this article can be found online at: http://journal.frontiersin.org/article/10.3389/fmicb. 2016.01666 


\section{REFERENCES}

Agarwal, K. L., Büchi, H., Caruthers, M. H., Gupta, N., Khorana, H. G., Kleppe, K., et al. (1970). Total synthesis of the gene for an alanine transfer ribonucleic acid from yeast. Nature 227, 27-34. doi: 10.1038/227027a0

Banks, G. R., Shelton, P. A., Kanuga, N., Holden, D. W., and Spanos, A. (1993). The Ustilago maydis narl gene encoding nitrate reductase activity: sequence and transcriptional regulation. Gene 131, 69-78. doi: 10.1016/0378-1119(93) 90670-X

Banno, I. (1967). Studies on the sexuality of Rhodotorula. J. Gen. Appl. Microbiol. 13, 167-196. doi: 10.2323/jgam.13.167

Barth, G. (1985). Genetic regulation of isocitrate lyase in the yeast Yarrowia lipolytica. Curr. Genet. 10, 119-124. doi: 10.1007/BF00636476

Brachman, C. B., Davies, A., Cost, G. J., Caputo, E., Li, J., Hieter, P., et al. (1998). Designer deletion strains derived from Saccharomyces cerevisiae S288C: a useful set of strains and plasmids for PCR-mediated gene disruption and other applications. Yeast 14, 115-132. doi: 10.1002/(SICI)10970061(19980130)14:2<115::AID-YEA204>3.0.CO;2-2

Buck, J. W., and Andrews, J. H. (1999). Attachment of the yeast Rhodosporidium toruloides is mediated by adhesives localized at sites of bud cell development. Appl. Environ. Microbiol. 65, 465-471.

Coelho, M. A., Almeida, J. M., Hittinger, C. T., and Goncalves, P. (2015). Draft genome sequence of Sporidiobolus salmonicolor CBS 6832, a redpigmented basidiomycetous yeast. Genome Announc. 3, e00444-15. doi: 10.1128/genomeA.00444-15

Duplessis, S., Cuomo, C. A., Lin, Y. C., Aerts, A., Tisserant, E., VeneaultFourrey, C., et al. (2011). Obligate biotrophy features unraveled by the genomic analysis of rust fungi. Proc. Natl. Acad. Sci. U.S.A. 108, 9166-9171. doi: 10.1073/pnas.1019315108

Evans, C. T., and Ratledge, C. (1984). Influence of nitrogen metabolism on lipid accumulation by Rhodosporidium toruloides CBS 14. J. Gen. Microbiol. 130, 1705-1710.

Firrincieli, A., Otillar, R., Salamov, A., Schmutz, J., Khan, Z., Redman, R. S., et al. (2015). Genome sequence of the plant growth promoting endophytic yeast Rhodotorula graminis WP1. Front. Microbiol. 6:978. doi: 10.3389/fmicb.2015.00978

Gebhart, D., Bahrami, A. K., and Sil, A. (2006). Identification of a copper-inducible promoter for use in ectopic expression in the fungal pathogen Histoplasma capsulatum. Eukaryot. Cell 5, 935-944. doi: 10.1128/EC.00028-06

Gelvin, S. B. (2006). "Agrobacterium virulence gene induction," in Methods in Molecular Biology: Agrobacterium Protocols, Vol. 343, 2nd edn, ed. K. Wang (Totowa, NJ: Humana Press), 77-84.

Gibson, D. G., Young, L., Chuang, R. Y., Venter, J. C., Hutchison, C. A. III, and Smith, H. O. (2009). Enzymatic assembly of DNA molecules up to several hundred kilobases. Nat. Methods 6, 343-345. doi: 10.1038/nmeth. 1318

Gilbert, H. J., and Tully, M. (1982). Synthesis and degradation of phenylalanine ammonia-lyase of Rhodosporidium toruloides. J. Bacteriol. 150, 498-505.

Grant, C. E., Bailey, T. L., and Noble, W. S. (2011). FIMO: scanning for occurrences of a given motif. Bioinformatics 27, 1017-1018. doi: 10.1093/bioinformatics/btr064

Grigoriev, I. V., Nikitin, R., Haridas, S., Kuo, A., Ohm, R., Otillar, R., et al. (2014). MycoCosm portal: gearing up for 1000 fungal genomes. Nucleic Acids Res. 42, D699-D704. doi: 10.1093/nar/gkt1183

Holsters, M., De Waele, D., Depicker, A., Messens, E., van Montagu, M., and Schell, J. (1978). Transfection and transformation of Agrobacterium tumefaciens. Mol. Gen. Genet. 163, 181-187. doi: 10.1007/BF00 267408

Huang, X.-F., Liu, J.-N., Lu, L.-J., Peng, K.-M., Yang, G.-X., and Liu, J. (2016). Culture strategies for lipid production using acetic acid as sole carbon source by Rhodosporidium toruloides. Bioresour. Technol. 206, 141-149. doi: 10.1016/j.biortech.2016.01.073

Kilaru, S., and Steinberg, G. (2015). Yeast recombination-based cloning as an efficient way of constructing vectors for Zymoseptoria tritici. Fungal Genet. Biol. 79, 76-83. doi: 10.1016/j.fgb.2015.03.017

Kleinegris, D. M., van Es, M. A., Janssen, M., Brandenburg, W. A., and Wijffels, R. H. (2010). Carotenoid fluorescence in Dunaliella salina. J. Appl. Phycol. 22, 645-649. doi: 10.1007/s10811-010-9505-y
Kosa, M., and Ragauskas, A. J. (2011). Lipids from heterotrophic microbes: advances in metabolism research. Trends Biotechnol. 29, 53-61. doi: 10.1016/j.tibtech.2010.11.002

Kumar, S., Kushwaha, H., Bachhawat, A. K., Raghava, G. P., and Ganesan, K. (2012). Genome sequence of the oleaginous red yeast Rhodosporidium toruloides MTCC 457. Eukaryot. Cell 11, 1083-1084. doi: 10.1128/EC. 00156-12

Labbé, S., and Thiele, D. J. (1999). Copper ion inducible and repressible promoter systems in yeast. Methods Enzymol. 306, 145-153. doi: 10.1016/S00766879(99)06010-3

Lee, J. J., Chen, L., Shi, J., Trzcinski, A., and Chen, W. N. (2014). Metabolomic profiling of Rhodosporidium toruloides grown on glycerol for carotenoid production during different growth phases. J. Agric. Food Chem. 62, 1020310209. doi: $10.1021 /$ jf502987q

Lin, X., Yang, F., Zhou, Y., Zhu, Z., Jin, G., Zhang, S., et al. (2012). Highlyefficient colony PCR method for red yeasts and its application to identify mutations within two leucine auxotroph mutants. Yeast 29, 467-474. doi: 10.1002/yea.2926

Lin, X. P., Wang, Y. N., Zhang, S. F., Zhu, Z. W., Zhou, Y. J. J., Yang, F., et al. (2014). Functional integration of multiple genes into the genome of the oleaginous yeast Rhodosporidium toruloides. FEMS Yeast Res. 14, 547-555. doi: 10.1111/1567-1364.12140

Liu, Y., Koh, C. M., Ngoh, S. T., and Ji, L. (2015). Engineering an efficient and tight D-amino acid-inducible gene expression system in Rhodosporidium/Rhodotorula species. Microb. Cell Fact. 14:170. doi: 10.1186/s12934-015-0357-7

Liu, Y., Koh, C. M., Sun, L., Hlaing, M. M., Du, M., Peng, N., et al. (2013). Characterization of glyceraldehyde-3-phosphate dehydrogenase gene RtGPD1 and development of genetic transformation method by dominant selection in oleaginous yeast Rhodosporidium toruloides. Appl. Microbiol. Biotechnol. 97, 719-729. doi: 10.1007/s00253-012-4223-9

Manetti, A. G., Rosetto, M., and Maundrell, K. G. (1994). nmt2 of fission yeast: a second thiamine-repressible gene co-ordinately regulated with nmt1. Yeast 10 , 1075-1082. doi: 10.1002/yea.320100809

Maundrell, K. (1990). nmt1 of fission yeast. A highly transcribed gene completely repressed by thiamine. J. Biol. Chem. 265, 10857-10864.

Menendez, J., Valdes, I., and Cabrera, N. (2003). The ICL1 gene of Pichia pastoris, transcriptional regulation and use of its promoter. Yeast 20, 1097-1108. doi: 10.1002/yea. 1028

Noskov, V. N., Karas, B. J., Young, L., Chuang, R. Y., Gibson, D. G., Lin, Y. C., et al. (2012). Assembly of large, high $\mathrm{G}+\mathrm{C}$ bacterial DNA fragments in yeast. ACS Synth. Biol. 1, 267-273. doi: 10.1021/sb3000194

Ouyang, S. Q., Beecher, C. N., Wang, K., Larive, C. K., and Borkovich, K. A. (2015). Metabolic impacts of using nitrogen and copper-regulated promoters to regulate gene expression in Neurospora crassa. G3 (Bethesda) 5, 1899-1908. doi: 10.1534/g3.115.020073

Punt, P. J., Dingemanse, M. A., Kuyvenhoven, A., Soede, R. D., Pouwels, P. H., and van den Hondel, C. A. (1990). Functional elements in the promoter region of the Aspergillus nidulans gpdA gene encoding glyceraldehyde-3phosphate dehydrogenase. Gene 93, 101-109. doi: 10.1016/0378-1119(90) 90142-E

Rennerfelt, E. (1937). Undersökningar över svampinfektionen i slipmassa och dess utveckling däri. Sven. Skogsvårdsföreningens Tidskr. 35, 43-159.

Ruff, J. A., Lodge, J. K., and Baker, L. G. (2009). Three galactose inducible promoters for use in C. neoformans var. grubii. Fungal Genet. Biol. 46, 9-16. doi: $10.1016 /$ j.fgb.2008.10.003

Sambrook, J., and Russell, D. W. (2001). Molecular Cloning: A Laboratory Manual. Cold Spring Harbor, NY: Cold Spring Harbor Laboratory.

Schweigert, N., Zehnder, A. J., and Eggen, R. I. (2001). Chemical properties of catechols and their molecular modes of toxic action in cells, from microorganisms to mammals. Environ. Microbiol. 3, 81-91. doi: 10.1046/j.14622920.2001.00176.x

Sidhu, Y. S., Cairns, T. C., Chaudhari, Y. K., Usher, J., Talbot, N. J., Studholme, D. J., et al. (2015). Exploitation of sulfonylurea resistance marker and nonhomologous end joining mutants for functional analysis in Zymoseptoria tritici. Fungal Genet. Biol. 79, 102-109. doi: 10.1016/j.fgb.2015.04.015

Siedenberg, D., Mestric, S., Ganzlin, M., Schmidt, M., Punt, P. J., van den Hondel, C., et al. (1999). GlaA promoter controlled production of a mutant 
green fluorescent protein (S65T) by recombinant Aspergillus niger during growth on defined medium in batch and fed-batch cultures. Biotechnol. Prog. 15, 43-50. doi: 10.1021/bp980105u

Singh, M. V., and Weil, P. A. (2002). A method for plasmid purification directly from yeast. Anal. Biochem. 307, 13-17. doi: 10.1016/S0003-2697(02) 00018-0

Solow, S. P., Sengbusch, J., and Laird, M. W. (2005). Heterologous protein production from the inducible MET25 promoter in Saccharomyces cerevisiae. Biotechnol. Prog. 21, 617-620. doi: 10.1021/bp049916q

Toome, M., Ohm, R. A., Riley, R. W., James, T. Y., Lazarus, K. L., Henrissat, B., et al. (2014). Genome sequencing provides insight into the reproductive biology, nutritional mode and ploidy of the fern pathogen Mixia osmundae. New Phytol. 202, 554-564. doi: 10.1111/nph.12653

Tully, M. (1985). Enrichment of mutants of Rhodosporidium toruloides by the use of inositol starvation. J. Basic Microbiol. 25, 683-686. doi: 10.1002/jobm.3620251020

van Larebeke, N., Engler, G., Holsters, M., van den Elsacker, S., Zaenen, J., Schilperoort, R. A., et al. (1974). Large plasmid in Agrobacterium tumefaciens essential for crown gall-inducing ability. Nature 252, 169-170. doi: $10.1038 / 252169 \mathrm{a} 0$

Wang, Q.-M., Yurkov, A. M., Göker, M., Lumbsch, H. T., Leavitt, S. D., Groenewald, M., et al. (2015). Phylogenetic classification of yeasts and related taxa within Pucciniomycotina. Stud. Mycol. 81, 149-189. doi: 10.1016/j.simyco.2015.12.002
Wang, Y., Lin, X., Zhang, S., Sun, W., Ma, S., and Zhao, Z. K. (2016). Cloning and evaluation of different constitutive promoters in the oleaginous yeast Rhodosporidium toruloides. Yeast 33, 99-106. doi: 10.1002/yea.3145

Wiebe, M. G., Koivuranta, K., Penttilä, M., and Ruohonen, L. (2012). Lipid production in batch and fed-batch cultures of Rhodosporidium toruloides from 5 and 6 carbon carbohydrates. BMC Biotechnol. 12:26. doi: 10.1186/1472-6750$12-26$

Zhang, S. Y., Skerker, J. M., Rutter, C. D., Maurer, M. J., Arkin, A. P., and Rao, C. V. (2016). Engineering Rhodosporidium toruloides for increased lipid production. Biotechnol. Bioeng. 113, 1056-1066. doi: 10.1002/bit.25864

Zhu, Z., Zhang, S., Liu, H., Shen, H., Lin, X., Yang, F., et al. (2012). A multi-omic map of the lipid-producing yeast Rhodosporidium toruloides. Nat. Commun. 3:1112. doi: $10.1038 /$ ncomms 2112

Conflict of Interest Statement: The authors declare that the research was conducted in the absence of any commercial or financial relationships that could be construed as a potential conflict of interest.

Copyright (C) 2016 Johns, Love and Aves. This is an open-access article distributed under the terms of the Creative Commons Attribution License (CC BY). The use, distribution or reproduction in other forums is permitted, provided the original author(s) or licensor are credited and that the original publication in this journal is cited, in accordance with accepted academic practice. No use, distribution or reproduction is permitted which does not comply with these terms. 